ARTICLE

Received 23 Jun 2015 | Accepted 10 Nov 2015 | Published 11 Dec $2015 \quad$ DOl: 10.1038/ncomms10170 OPEN

\title{
Efficient quasi-monoenergetic ion beams from laser-driven relativistic plasmas
}

Sasi Palaniyappan ${ }^{1}{ }^{\star}$, Chengkun Huang ${ }^{1, \star}$, Donald C. Gautier ${ }^{1}$, Christopher E. Hamilton $^{1}$, Miguel A. Santiago ${ }^{1}$, Christian Kreuzer ${ }^{2}$, Adam B. Sefkow ${ }^{3}$, Rahul C. Shah ${ }^{1} \&$ Juan C. Fernández ${ }^{1}$

Table-top laser-plasma ion accelerators have many exciting applications, many of which require ion beams with simultaneous narrow energy spread and high conversion efficiency. However, achieving these requirements has been elusive. Here we report the experimental demonstration of laser-driven ion beams with narrow energy spread and energies up to $18 \mathrm{MeV}$ per nucleon and $\sim 5 \%$ conversion efficiency (that is $4 \mathrm{~J}$ out of $80-\mathrm{J}$ laser). Using computer simulations we identify a self-organizing scheme that reduces the ion energy spread after the laser exits the plasma through persisting self-generated plasma electric $\left(\sim 10^{12} \mathrm{Vm}^{-1}\right)$ and magnetic $\left(\sim 10^{4} \mathrm{~T}\right)$ fields. These results contribute to the development of next generation compact accelerators suitable for many applications such as isochoric heating for ion-fast ignition and producing warm dense matter for basic science.

\footnotetext{
${ }^{1}$ Los Alamos National Laboratory, Los Alamos, New Mexico 87545, USA. ${ }^{2}$ Ludwig-Maximilian-University, Munich, Germany. ${ }^{3}$ Sandia National Laboratory, Albuquerque, New Mexico 87185, USA. * These authors contributed equally to this work. Correspondence and requests for materials should be addressed to S.P. (email: sasi@lanl.gov) or to C.H. (email: huangck@lanl.gov).
} 
aser-driven ion beams with narrow energy spread and high conversion efficiency would be transformational because they could deliver unprecedented power densities. For example, warm dense matter with conditions relevant to stars and planetary cores can be created in the laboratory by isochoric heating of bulk matter with such ion beams ${ }^{1,2}$. Ion-fast ignition is an extreme example of isochoric heating, where laserdriven ion beams can ignite compressed fuel to generate fusion energy $^{3-7}$.

Despite a decade-plus effort, achieving laser-driven ion beams with simultaneous narrow energy spread and high efficiency remains a big challenge ${ }^{8-20}$. Widely explored schemes for laser-driven ion beam generation include target normal sheath acceleration (TNSA) $)^{9-11,21-29}$, radiation pressure acceleration (RPA) ${ }^{12-14,20,30-33}$, coherent acceleration of ions by laser $(\mathrm{CAIL})^{34,35}$, breakout afterburner $(\mathrm{BOA})^{36-38}$, magnetic vortex acceleration (MVA) $)^{39-42}$ and collisionless shock acceleration $(\mathrm{CSA})^{16,17}$. Broadly, these attempts can be classified into three categories, viz., (1) picosecond laser pulses interacting with microns-thick opaque targets, mostly TNSA, (2) ultrashort femtosecond laser pulses interacting with either ultrathin solid targets such as RPA and CAIL or near-critical/gas targets such as MVA and (3) the intermediate regime of picosecond laser pulses interacting with either thin sub-micron solid targets in the relativistic transparency (RT) regime such as BOA, or gas targets such as CSA.

The TNSA mechanism typically produces a broad exponential ion energy spectrum ${ }^{21-27,29}$. In 2006, Schwoerer et al. ${ }^{10}$ reported $1.2 \mathrm{MeV}$ quasi-monoenergetic proton beam with $\sim 40 \%$ energy spread and Hegelich et al. ${ }^{11}$ reported $3 \mathrm{MeV}$ per nucleon quasi-monoenergetic carbon ion beam with $17 \%$ energy spread, both under TNSA scheme using complex engineered targets with thin coating on the rear side (category 1). Nevertheless their conversion efficiency was much $<1 \%$ (refs 9-11). In 2009, Henig et al. ${ }^{13}$ reported quasi-monoenergetic $2.5 \mathrm{MeV}$ per nucleon carbon ion beam with $\sim 35 \%$ energy spread and $2.5 \%$ conversion efficiency (carbon ion beam contained $35 \mathrm{~mJ}$ of energy) when a 30-TW Ti:Sapphire ultrashort laser pulse interacted with a 5-nmthick diamond-like carbon foil (category 2). In 2011, Haberberger et al. ${ }^{16}$ reported quasi-monoenergetic $20 \mathrm{MeV}$ protons with $1 \%$ energy spread and efficiency much $<1 \%$ when multi-TW Neptune $\mathrm{CO}_{2}$ laser pulses interacted with a hydrogen gas jet (category 3). In 2012, Kar et al. ${ }^{14}$ reported quasi-monoenergetic $\sim 7 \mathrm{MeV}$ per nucleon carbon ion beam with $\sim 60 \%$ energy spread and $1 \%$ conversion efficiency when a $0.25 \mathrm{PW}$ Vulcan picosecond laser pulse $(\sim 200 \mathrm{~J}, \sim 800 \mathrm{fs})$ interacted with a 50 -nm-thick copper foil (category 3 ). These results illustrate the tremendous progress in generating efficient quasi-monoenergetic ion beams from laser-driven plasmas. However, applications such as ion-fast ignition require quasi-monoenergetic ion beams at tens of $\mathrm{MeV} /$ nucleon with efficiency $\sim 10 \%$.

Here we report ion beams with narrow spectral peaks at energies up to $310 \mathrm{MeV}$ for $\mathrm{Al}^{11+}(11.5 \mathrm{MeV}$ per nucleon) and $220 \mathrm{MeV}$ for $\mathrm{C}^{6+}(18.3 \mathrm{MeV}$ per nucleon) with $\sim 5 \%$ conversion efficiency from relativistic laser-plasma interaction in category 3. Also, we demonstrate that by increasing the focused laser intensity fourfold (by reducing the focusing optic $f$-number twofold), the spectral-peak energy increases twofold. These results are obtained with the $0.12 \mathrm{PW}(80 \mathrm{~J}, 650 \mathrm{fs}$, linear polarized) Trident laser ${ }^{43}$ at the Los Alamos National Laboratory, irradiating planar foils of an optimized thickness of up to $250 \mathrm{~nm}$. Although the experimental set-up here is similar to prior ones at Trident laser facility, the new results demonstrated here are primarily due to reduced target pre-expansion and optimization of the onset of relativistic transparency in dense target plasmas that enables (according to simulation) a self-organized process which reduces the ion beam energy spread (discussed later).

\section{Results}

Overview. Table 1 summarizes the laser/target parameters along with the resulting plasma and ion beam properties for seven different sets of experiments (rows 2-8) discussed in this article. Experiments I, II, IV, V and VII indicate the conditions under which ion spectral peak is generated.

\section{Quasi-monoenergetic ions from relativistically transparent plasmas} Figure 1 shows schematic layout of the experiment. An $f / 3$ offaxis parabola focuses the $80 \mathrm{~J}, 0.12 \mathrm{PW}$ Trident laser (peak laser intensity of $2 \times 10^{20} \mathrm{~W} \mathrm{~cm}^{-2}$ ) onto the target, such as a 110 nm-thick aluminium foil (density-2.7 $\mathrm{g} \mathrm{cm}^{-3}$ ) (Table $1-$ experiment I). Multiple optical and particle diagnostics were used to characterize the experiment. The sharp drop in reflected laser-light intensity (Fig. 1a) near the peak of the main laser pulse indicates the ensuing relativistic transparency phase in the laser-plasma interaction. The temporal phase of the transmitted

Table 1 | Laser/target parameters and properties of generated ion beams and plasmas.

\begin{tabular}{|c|c|c|c|c|c|c|c|c|}
\hline Experiment. & $\begin{array}{l}\text { Laser } \\
\text { focus }\end{array}$ & $\begin{array}{l}\text { Intensity } \\
\left(\mathbf{W} \mathbf{c m}^{-2}\right)\end{array}$ & Target ${ }^{\star}$ & $v_{c s}\left(\mu \mathrm{m} \mathrm{ps}^{-1}\right)^{\dagger}$ & $\begin{array}{l}\text { Ion spectral } \\
\text { peak }^{\ddagger}\end{array}$ & $\begin{array}{l}\text { FWHM Energy } \\
\text { spread } \ddagger^{\ddagger}(\%)\end{array}$ & $\begin{array}{l}\text { Conversion } \\
\text { efficiency (\%) }\end{array}$ & $\begin{array}{c}\text { Proton spectral } \\
\text { peak }^{\ddagger}(\mathrm{MeV})\end{array}$ \\
\hline I & $f / 3$ & $2 \times 10^{20}$ & 110 nm Al & 1.7 & $\begin{array}{l}165 \mathrm{MeV} \mathrm{Al}{ }^{11+} \\
(6.1 \mathrm{MeV} / \mathrm{u})\end{array}$ & 7 & 5 & Below TP cut-off \\
\hline II & $f / 3$ & $2 \times 10^{20}$ & $\begin{array}{l}110 \mathrm{~nm} \mathrm{Al} / \\
10 \mathrm{~nm} \mathrm{C}\end{array}$ & - & $\begin{array}{l}80 \mathrm{MeV} \mathrm{C}^{6+} \\
(6.7 \mathrm{MeV} / \mathrm{u})\end{array}$ & 15 & - & Below-TP cut-off \\
\hline III & $f / 3$ & $2 \times 10^{20}$ & $100 \mathrm{~nm} \mathrm{C}$ & $0.6^{\S}$ & No spectral peakll & - & - & No spectral peakll \\
\hline IV & $f / 1.5$ & $8 \times 10^{20}$ & $250 \mathrm{~nm} \mathrm{Al}$ & 1.1 & $\begin{array}{l}310 \mathrm{MeV} \mathrm{Al}{ }^{11+} \\
(11.5 \mathrm{MeV} / \mathrm{u})\end{array}$ & 41 & 4 & 12.6 \\
\hline V & $f / 1.5$ & $8 \times 10^{20}$ & $\begin{array}{l}250 \mathrm{~nm} \mathrm{Al} / \\
10 \mathrm{~nm} \mathrm{C}\end{array}$ & - & $\begin{array}{l}120 \mathrm{MeV} \mathrm{C}^{6+} \\
(10 \mathrm{MeV} / \mathrm{u})\end{array}$ & 54 & - & 17 \\
\hline VI & $f / 1.5$ & $8 \times 10^{20}$ & $250 \mathrm{~nm} \mathrm{C}$ & -0.46 & No spectral peak & - & - & No spectral peak \\
\hline VII & $f / 1.5$ & $6 \times 10^{20}$ & $250 \mathrm{~nm} \mathrm{C}$ & 1.2 & $\begin{array}{l}220 \mathrm{MeV} \mathrm{C} \mathrm{C}^{6+} \\
(18.3 \mathrm{MeV} / \mathrm{u})\end{array}$ & 23 & 4 & 23.3 \\
\hline \multicolumn{9}{|c|}{ 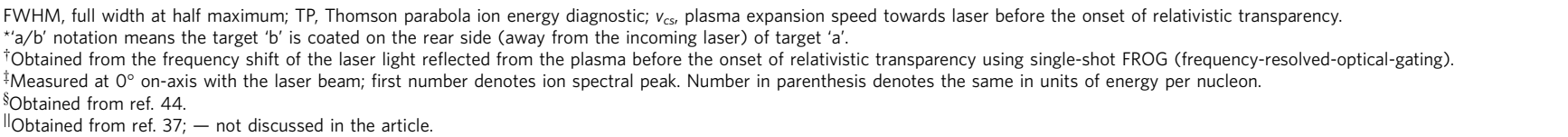 } \\
\hline
\end{tabular}



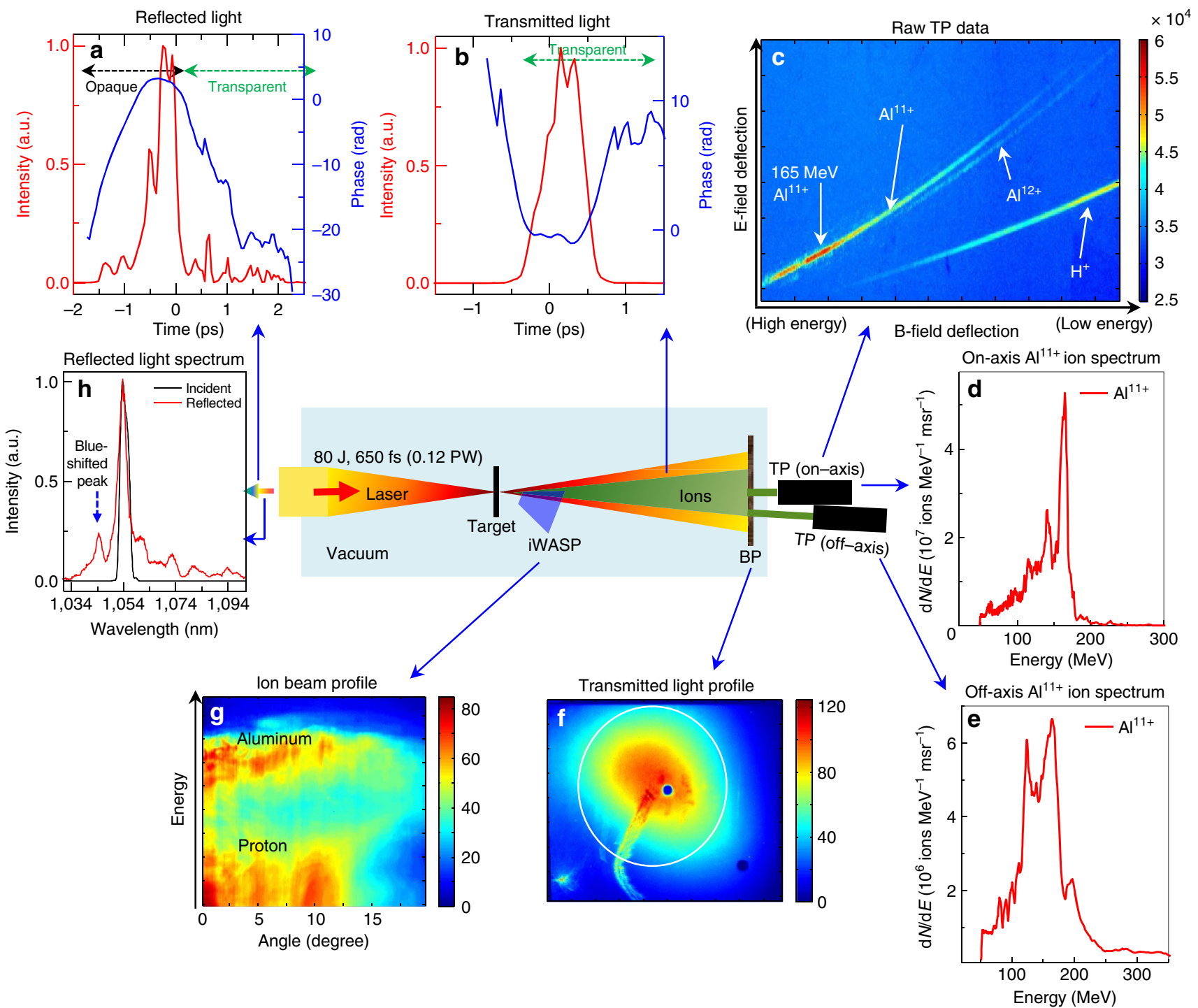

Figure 1 | Schematic of experimental set-up (f/3 laser focus onto $\mathbf{1 1 0} \mathbf{~ n m ~ A l ~ f o i l ) . ~ T h e ~} 0.12 \mathrm{PW}$ Trident laser is focused with $f / 3$ off-axis parabola (peak intensity $-2 \times 10^{20} \mathrm{~W} \mathrm{~cm}^{-2}$ ) onto a 110-nm-thick aluminium foil (Table 1-Experiment I). (a) Time-resolved reflected light intensity (solid red line) and its temporal phase (solid blue line) measured using a single-shot frequency-resolved-optical-gating (FROG) showing the onset of relativistic transparency. The positive (negative) slope of the temporal phases indicate spectral blue (red) shift via $\omega_{t}=\mathrm{d} \phi / \mathrm{d} t$, where $\omega_{t}$ is the instantaneous angular frequency; (b) time-resolved transmitted light intensity (solid red line) and its temporal phase (solid blue line) measured using a separate FROG showing the phase reversal during relativistic transparency; (c) raw Thomson parabola (TP) data; (d) measured $\mathrm{Al}^{11+}$ ion spectrum on-axis (solid red line) with $166 \mathrm{MeV}$ spectral peak; (e) measured $\mathrm{Al}^{11+}$ ion spectrum $8.5^{\circ}$ off-axis (solid red line) with $165 \mathrm{MeV}$ spectral peak. TP used image plate (IP) detectors crosscalibrated against CR-39 nuclear track detectors. The TP instrumental cut-off is at $50 \mathrm{MeV}$ due to an 18 - $\mu \mathrm{m}$-thick aluminium foil placed in front of the ion detector to block laser light; $(\mathbf{f})$ transmitted laser beam profile captured on a Macor plate $(30.5 \times 30.5 \mathrm{~cm}$ square $)$ and imaged onto a separate CCD camera (Apogee Alta U8300). This is used to quantify the amount of laser energy transmitted through the plasma; ( $\mathbf{g}$ ) angularly resolved ion energy spectra measured using an ion wide-angle magnetic spectrometer (iWASP); (h) reflected light spectrum (solid red line) measured using an infra-red spectrometer (Bruker Optics with Andor iDUS $1.7 \mu \mathrm{m}$ InGaAs CCD) along with incident laser spectrum (solid black line) show a blue-shifted spectral peak.

laser light (Fig. 1b) indicates the evolution of the electron density and relativistic $\gamma$ factor during relativistic transparency phase consistent with earlier results ${ }^{44}$. Since the density is monotonically decreasing, the temporal phase reversal reflects the peaking of $\gamma$, in agreement with simulation. In these shots, the plasma turned relativistically transparent near the peak of the laser pulse. Empirically this appears to be a necessary condition to realize accelerated ion spectral peaks with the Trident laser pulse. The transmitted laser beam (Fig. 1f) contains $20 \%$ of the incident laser energy (that is $16 \mathrm{~J}$ ). Relativistic transparency enables strong volumetric laser coupling into the dense plasma.
The time-integrated reflected light spectrum (Fig. 1h) from the aluminium foil shows a $9-\mathrm{nm}$ blue-shifted peak at $1,044 \mathrm{~nm}$. This Doppler shift $\mathrm{d} \lambda / \lambda=2 v / c \cong-0.009$ indicates that the plasma critical surface expands at a speed of $1.4 \mu \mathrm{mps}^{-1}$ towards the incoming laser. The Doppler shift in the temporal phase of the backscattered FROG measurement provides the instantaneous velocity of the plasma critical surface $\left(v_{c s}\right)$ at the front side of the plasma $\left(\mathrm{d} \lambda / \lambda=2 v_{c s} / c=-\mathrm{d} \omega / \omega \stackrel{\text { yields }}{\rightarrow} v_{c s}(t)=-\frac{c}{2 \omega} \frac{\mathrm{d} \phi}{\mathrm{d} t}\right)$. The time-resolved temporal phase of the reflected light from aluminium (Fig. 1a) shows an early positive phase slope (that is 
frequency blue-shift) $\mathrm{d} \omega / \omega=0.011$ from $t=-1.75 \mathrm{ps}$ to $t=-0.75 \mathrm{ps}$. This corresponds to a plasma critical surface expansion of $1.7 \mu \mathrm{m} \mathrm{ps}^{-1}$ towards the laser. It is important to remark that the backscattered light from the laser-pulse pedestal at the nanosecond timescale is too dim, and therefore these diagnostics are only sensitive to backscattered light during the rising portion of the main pulse. The plasma expansion velocity during the main laser pulse is inversely correlated to the target pre-expansion during the nanosecond pedestal. This is discussed in detail later under the section 'Implication of backscatteredlaser measurements'.

The raw Thomson parabola (TP) data (Fig. 1c) show the dominant $\mathrm{Al}^{11+}$ ion and proton impurity traces along with a faint trace of $\mathrm{Al}^{12+}$. An atomic ionization calculation of aluminium for Trident laser parameters ( $f / 3$ focus) shows the aluminium is ionized to $\mathrm{Al}^{11+}$ and there is barely any $\mathrm{Al}^{12+}$ due to the huge inner-shell gap between the respective ionization potentials (Supplementary Fig. 1) ${ }^{45}$. The $\mathrm{Al}^{11+}$-ion energy spectrum measured on-axis (Fig. 1d) peaks at $166 \mathrm{MeV}$ (6.2 MeV per nucleon) with a $7 \%$ energy spread. The integrated spectrum yields a total of $1.8 \times 10^{9}$ ions per millisteradian (msr) with average energy of $123 \mathrm{MeV}$. The corresponding spectrum measured at $8.5^{\circ}$ off-axis (Fig. 1e), for another shot with similar target and laser conditions, shows a similar ion peak at $165 \mathrm{MeV}$ with $30 \%$ energy spread (total of $5.5 \times 10^{8}$ ions per msr with average energy of $131 \mathrm{MeV}$ ). The proton spectrum has no spectral peak within the 9 to $50 \mathrm{MeV}$ range (Supplementary Fig. 2). TP design and settings (the short gap between the electrodes and the high applied voltage) precluded measuring protons below $9 \mathrm{MeV}$. The data set associated with single-shot high-power laser systems with a long-laser cool-down period $(\sim 1.5 \mathrm{~h})$ is necessarily small. Supplementary Figure 3 shows measured off-axis $\mathrm{Al}^{11+}$ ion spectra for a series of five shots that illustrate the shot-to-shot variation in the results. We estimate a total of $2 \times 10^{11} \mathrm{Al}^{11+}$ ions in the full beam (average energy of $127 \mathrm{MeV}$; see Methods for details on conversion efficiency estimation). This result implies the aluminium ion beam contains $4 \mathrm{~J}$ energy out of the $80 \mathrm{~J}$ incident laser energy ( $5 \%$ conversion efficiency) and $0.35 \mu \mathrm{C}$ of charge. A similar calculation for protons yields $0.6 \%$ conversion efficiency.

The charge-to-mass ratios of $\mathrm{Al}^{11+}(0.407)$ and $\mathrm{C}^{5+}(0.417)$ are close. Therefore conceivably the $\mathrm{Al}^{11+}$ trace in the TP data could be contaminated by $\mathrm{C}^{5+}$ from hydrocarbon contamination of aluminium foils. To address that concern, we deliberately coated the rear side of $110-\mathrm{nm}$ aluminium targets with a $10 \mathrm{~nm}$ of carbon layer to mimic such contamination and repeated the same experiment (Table 1 -experiment II). Figure 2a shows the raw TP data from that target. The dominant trace is $\mathrm{C}^{6+}$, not $\mathrm{C}^{5+}$. Therefore, it seems likely that $\mathrm{C}$, if present as a contaminant in the Al target data in Fig. 1c, then would show up as $C^{6+}$, clearly distinguishable from the $\mathrm{Al}^{11+}$ trace in the $\mathrm{TP}$, rather than a contributor to it as $\mathrm{C}^{5+}$. The $\mathrm{C}^{6+}$ trace in Fig. $2 \mathrm{~b}$ from the layered target shows a spectral peak around $80 \mathrm{MeV}$ (6.7 MeV per nucleon) similar to the $6.2 \mathrm{MeV}$ per nucleon $\mathrm{Al}^{11+}$ ion spectral peak from a pure aluminium target. This suggests that the underlying laser-plasma dynamics responsible for ion spectral peak generation operate at the rear of the target, and are robust and transferable to other ion species with proper optimization. The protons again show no spectral peak above $9 \mathrm{MeV}$ (Fig. 2c).

Prior experiments with a similar set-up using synthetic diamond targets ${ }^{46}$ did not generate quasi-monoenergetic ion beams (Table 1 -experiment III) ${ }^{37}$ as seen here with aluminium. This difference is explained later under the section 'Implication of backscattered-laser measurements'.

Scaling to higher laser intensity. The Trident laser was focused with a faster $f / 1.5$ off-axis parabola, creating a peak laser intensity of $8 \times 10^{20} \mathrm{~W} \mathrm{~cm}^{-2}(4 \times$ the $\mathrm{f} / 3$ intensity) onto a 250 -nm-thick aluminium foil (Table 1-Experiment IV). The target thickness was chosen to keep the onset of relativistic transparency near the temporal peak of the laser pulse. Here two newly developed high-dispersion TPs provided simultaneous on-axis and $11^{\circ}$ off-axis ion spectral measurements (see Methods and Supplementary Fig. 4). The raw TP data (Fig. 3a) shows well-separated traces of $\mathrm{Al}^{11+}, \mathrm{Al}^{12+}, \mathrm{Al}^{13+}$ and protons. The $\mathrm{Al}^{11+}$ trace is still dominant. The on-axis $\mathrm{Al}^{11+}$ ion spectrum (red solid line in Fig. 3b) peaks at $310 \mathrm{MeV}(11.5 \mathrm{MeV}$ per nucleon) with $41 \%$ energy spread (total of $4.2 \times 10^{8}$ ions per msr with average energy of $179 \mathrm{MeV}$ ). The simultaneous off-axis $\mathrm{Al}^{11+}$ spectrum (solid blue line in Fig. 3b) peaks at $250 \mathrm{MeV}$ (9.3 MeV per nucleon) with $21 \%$ energy spread (total of $4.7 \times 10^{8}$ ions per msr with average energy of $167 \mathrm{MeV})$. The proton spectra (Fig. 3c) peaks around $12 \mathrm{MeV}$, a similar energy per nucleon as the $\mathrm{Al}^{11+}$.

The ion/proton beam profile (Fig. 3d) shows the ion beam extending up to a $28^{\circ}$ FWHM. Assuming radial symmetry, we estimate a total of $1 \times 10^{11} \mathrm{Al}^{11+}$ ions in the full beam (average energy of $173 \mathrm{MeV}$ ). This implies a $\sim 3-\mathrm{J}$ aluminium beam out of the $80 \mathrm{~J}$ incident laser energy (4\% conversion efficiency). A similar calculation for protons yields $\sim 0.2 \%$ conversion efficiency. This interaction also exhibits plasma expansion towards the laser of $1.1 \mu \mathrm{mps}^{-1}$ derived from the reflected FROG measurement (Fig. 3e), and $1.2 \mu \mathrm{mps}^{-1}$ from the reflected spectral peak blue-shifted by $8 \mathrm{~nm}$ (Fig. $3 \mathrm{f}$ ), similar to the results with $f / 3$ and $110 \mathrm{~nm} \mathrm{Al}$.

Once again, we address the concern about possible hydrocarbon contamination of the 'pure' Al nanofoil targets by comparing with the results from $\mathrm{Al}$ nanofoils coated with $10 \mathrm{~nm}$ of carbon layer on the rear side (Table 1-Experiment V).
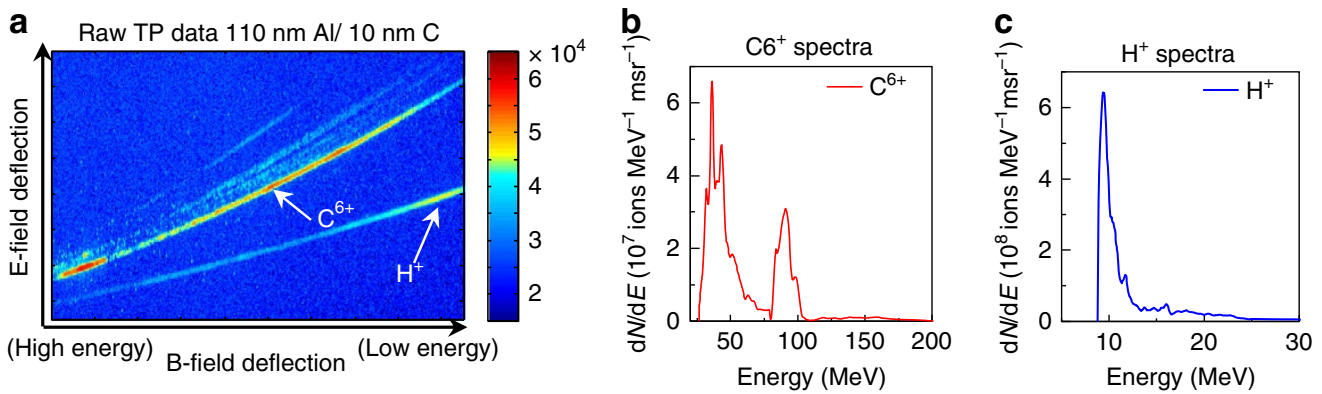

Figure 2 | TP results from f/3 laser focus interaction with $\mathbf{1 0} \mathbf{n m}$ carbon coated $\mathbf{1 1 0} \mathbf{~ n m ~ A l ~ f o i l . ~ ( a - c ) ~ ( T a b l e ~ 1 - E x p e r i m e n t ~ I I ) ~ ( a ) ~ R a w ~ T P ~ d a t a ~ f r o m ~}$ $110 \mathrm{~nm} \mathrm{Al} / 10 \mathrm{~nm} C$ foil showing pre-dominant $C^{6+}$ trace; (b) measured on-axis $C^{6+}$ ion spectrum (solid red line); (c) measured on-axis proton spectrum (solid blue line). 

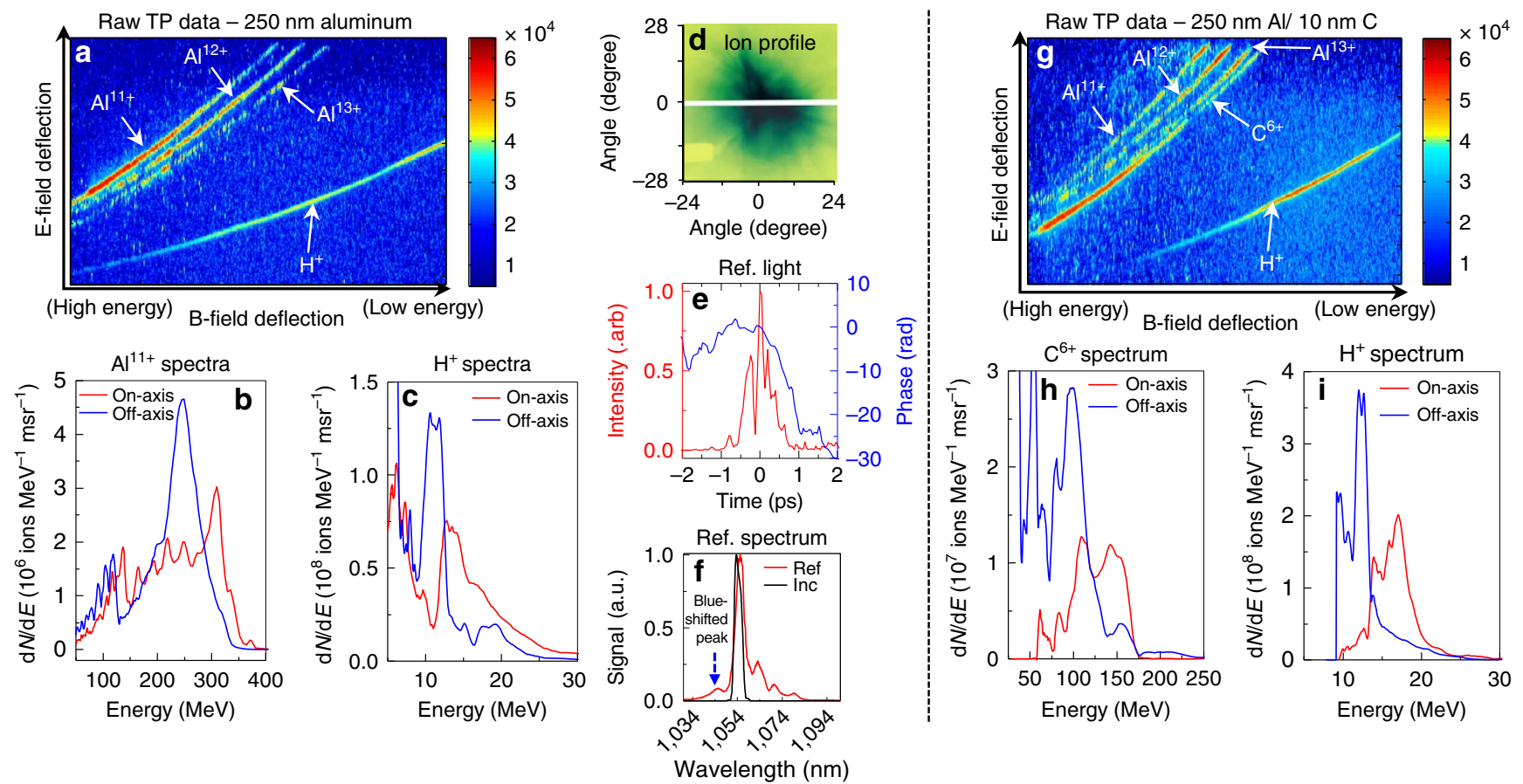

Figure 3 | f/1.5 laser focus interaction with aluminium foil. (a) Raw TP data from f/1.5 laser focus interaction with the 250-nm Al foil (Table 1Experiment IV); (b) spectrally peaked $\mathrm{Al}^{11+}$ spectra on-axis (solid red line) and $11^{\circ}$ off-axis (solid blue line); (c) spectrally peaked proton spectra on-axis (solid red line) and $11^{\circ}$ off-axis (solid blue line); (d) ion/proton beam profile measured using radiochromic film (RCF); (e) time-resolved reflected light intensity (solid red line) and temporal phase (solid blue line) indicative of relativistic transparency; (f) time-integrated reflected light spectrum (solid red line) along with incident light spectrum (solid black line); (g) raw TP data from $f / 1.5$ laser focus interaction with the $10 \mathrm{~nm}$ carbon coated on the rear side of the 250-nm-thick Al foil (Table 1-Experiment V); (h) spectrally peaked $\mathrm{C}^{6+}$ spectra measured on-axis (solid red line) and $11^{\circ}$ off-axis (solid blue line); (i) corresponding spectrally peaked proton spectra measured on-axis (solid red line) and $11^{\circ}$ off-axis (solid blue line).

The raw TP data from these targets (Fig. 3g) again shows predominantly $\mathrm{C}^{6+}$. The $\mathrm{C}^{6+}$ spectrum (Fig. $3 \mathrm{~h}$ ) peaks around $120 \mathrm{MeV}$ (10 MeV per nucleon) measured on-axis, and around $100 \mathrm{MeV}$ (8.3 MeV per nucleon) measured off-axis, similar to the $\mathrm{Al}^{11+}$ energy/nucleon spectral peaks from pure aluminium targets. The proton spectrum from the carbon coated aluminium foil (Fig. 3i) peaks at $18 \mathrm{MeV}$ (on-axis) and $12 \mathrm{MeV}$ (off-axis).

Implication of backscattered-laser measurements. When using the same experimental set-up, the 'Al' target produces an ion spectral peak (Table 1-Experiment I), but the 'C' does not (Table 1-Experiment III). Here we compare the backscatteredlasers results for experiments I and III ( $f / 3$ laser focus interacting with $110 \mathrm{~nm} \mathrm{Al}$ and $100 \mathrm{~nm} \mathrm{C}$ targets, respectively) to explain the differences in their performance. A notable difference is that the backscattered-laser spectrum from ' $C$ ' lacks a blue-shifted spectral peak (see Fig. $4 \mathrm{~b}$ inset in ref. 44). Also, the time-dependent reflected laser from diamond (see Fig. $4 \mathrm{~b}$ in ref. 44) shows an early frequency blue-shift of $\mathrm{d} \omega / \omega=0.004$ (plasma expansion of $0.6 \mu \mathrm{m} \mathrm{ps}^{-1}$ towards the incoming laser)-nearly three times slower compared to aluminium $\left(1.7 \mu \mathrm{mps}^{-1}\right.$-Experiment I). Radiation-hydrodynamics simulations indicate, perhaps counterintuitively, that the expansion velocity during the long pedestal, which we cannot measure, is inversely correlated to the expansion velocity during the rising edge of the short-pulse, which we measure, as discussed below.

Figure 4a,b show results from two-dimensional (2D) radiation-hydrodynamics simulations of the pre-expansion of 110 -nm-thick ' $\mathrm{Al}$ ' and ' $\mathrm{C}$ ' foils from the Trident laser pedestal using the HYDRA code ${ }^{47}$ (see Supplementary Fig. 5 for the laser contrast). The HYDRA code has been recently benchmarked
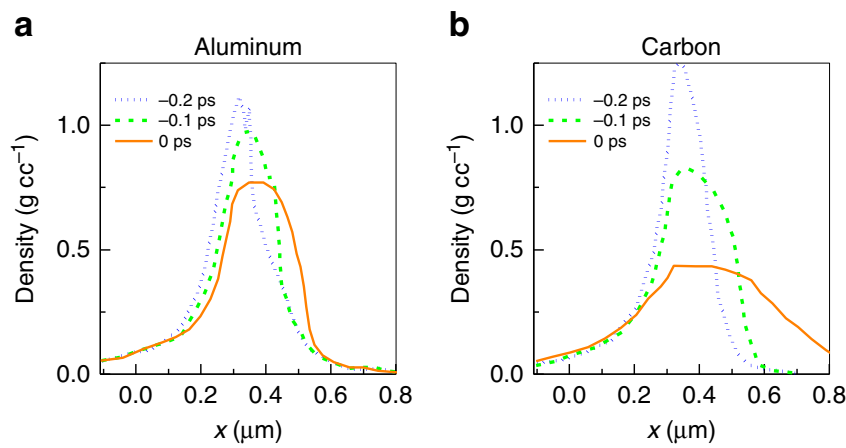

Figure 4 | Laser pedestal pre-expansion of targets. Results from 2D rad-hydro HYDRA simulation of Trident laser pedestal ( $f / 3$ focus) preexpansion of 110-nm-thick aluminium (a) and carbon (b) targets at $-0.2 \mathrm{ps}$ (blue dotted line), $-0.1 \mathrm{ps}$ (green dashed line) and $0 \mathrm{ps}$ (orange solid line) before the end of the rad-hydro simulation. The targets were initially located from $x=-0.11 \mu \mathrm{m}$ to $x=0 \mu \mathrm{m}$.

against pre-expansion measurements of TNSA targets at the Trident facility ${ }^{29}$. Both the targets have initial density of $2.7 \mathrm{~g} \mathrm{~cm}^{-3}$. When the laser pedestal initially shocks the targets 'Al' begins to disassemble faster than ' $\mathrm{C}$ ' (not shown). But as the shock passes through the targets ' $\mathrm{C}$ ' falls apart about twice as fast as 'Al' (Fig. 4a,b). At the end of the simulations, the peak carbon density $\left(0.4 \mathrm{~g} \mathrm{~cm}^{-3}\right)$ is a factor of two lower than the peak aluminium density $\left(0.8 \mathrm{~g} \mathrm{~cm}^{-3}\right)$ when expanded by the same laser pedestal. Later, when the main pulse arrives at the plasma, it heats the plasma rapidly and consequently the plasma expansion speeds up. At the same time, the laser radiation pressure can now 
reduce the plasma expansion speed towards the laser (and subsequently turn it around) more effectively in carbon plasma compared with the aluminium plasma because of its lower plasma density, consistent with the backscattered FROG measurements.

Overall, these results suggest two crucial elements in our experiments that result in ion spectral peaks viz., (1) reducing pre-plasma expansion to maintain higher initial plasma density and (2) ensuring the onset of RT occurs near the peak of the laser pulse. Our hypothesis is that lower 'initial' plasma density leads to lower residual self-generated plasma fields, too weak to reduce the ion energy spread after the laser-plasma interaction endssupported by the particle-in-cell simulations discussed later. The above discussion was successfully put to the test by generating a quasi-monoenergetic $\mathrm{C}$ peak at higher intensity as discussed below.

Optimization of laser interaction with carbon foils. Compared with the f/1.5-aluminium experiment (Table 1-Experiment IV), a similar laser interaction with a 250-nm-thick diamond foil (Table 1-Experiment VI) did not generate $\mathrm{C}^{6+}$ or proton spectral peaks (blue solid line in Fig. 5b,c, respectively). The early plasma dynamics of 'C' (Table 1-Experiment VI) differ significantly from the 'Al' (Table 1-Experiment IV) - the reflected light lacks both the initial frequency blue-shift (blue solid line in Fig. 5e) and the blue spectral peak (solid red line in Fig. 5d). Also the main reflected light peak is red shifted by $4.5 \mathrm{~nm}$ (solid red line in Fig. 5d), compared with only $1.7 \mathrm{~nm}$ for aluminium (solid red line in Fig. 3f). As above, these results indicate that the ' $C$ ' target disassembles faster than 'Al' during the ns laser pedestal resulting in a much lower ' $\mathrm{C}$ ' peak plasma density than 'Al' when the main laser-pulse arrives. Lower plasma density enables the main laser-pulse to push the plasma away from the laser instantly and causes the lack of initial frequency blue-shift and the blue spectral peak in the backscattered-laser measurements of ' $\mathrm{C}$ ' that are seen in 'Al'.

Understanding the significance of target pre-expansion, we have taken a different tack with synthetic diamond-foils to match their pre-expansion and early-expansion dynamics to that of ' $\mathrm{Al}$ ' foil with a view in achieving a $\mathrm{C}$-ion spectral peak. We have no benchmarked way to decrease the Trident pedestal contrast any further. To reduce the diamond foil pre-expansion, we simply reduced the incident laser energy from 80 to $60 \mathrm{~J}$, which correspondingly reduced the pedestal and main pulse intensities by $25 \%$ (Table 1 -Experiment VII). With reduced laser energy, the reflected light shows a ' $C$ ' foil expansion very similar to the aluminium foil interaction discussed earlier-early frequency blue-shift of $\mathrm{d} \omega / \omega=0.008$ (solid blue line in Fig. $5 \mathrm{f}$ ) that is a plasma expansion of $1.2 \mu \mathrm{mps}^{-1}$ towards the laser and a $8-\mathrm{nm}$ blue-spectral peak in the reflected light (solid blue line in Fig. $5 \mathrm{~d}$ ). The corresponding raw TP data is shown in Fig. 5a. The measured on-axis $\mathrm{C}^{6+}$ ion spectrum (solid red line in Fig. 5b) is peaked at $220 \mathrm{MeV}$ (18.3 MeV per nucleon). The simultaneous off-axis spectrum (solid green line in Fig. 5b) is double peaked at $106 \mathrm{MeV}$ (8.8 MeV per nucleon) and at $150 \mathrm{MeV}(12.5 \mathrm{MeV}$ per nucleon). The corresponding proton spectrum peaks at $23.3 \mathrm{MeV}$ measured on-axis (solid red line in Fig. 5c) and $17.8 \mathrm{MeV}$ measured off-axis (solid green line in Fig. 5c). The same estimation as before yields a $\sim 4 \%$ conversion efficiency (total of $2 \times 10^{11}$ ions, $81 \mathrm{MeV}$ average energy) for $\mathrm{C}^{6+}$ and $0.6 \%$ for protons.
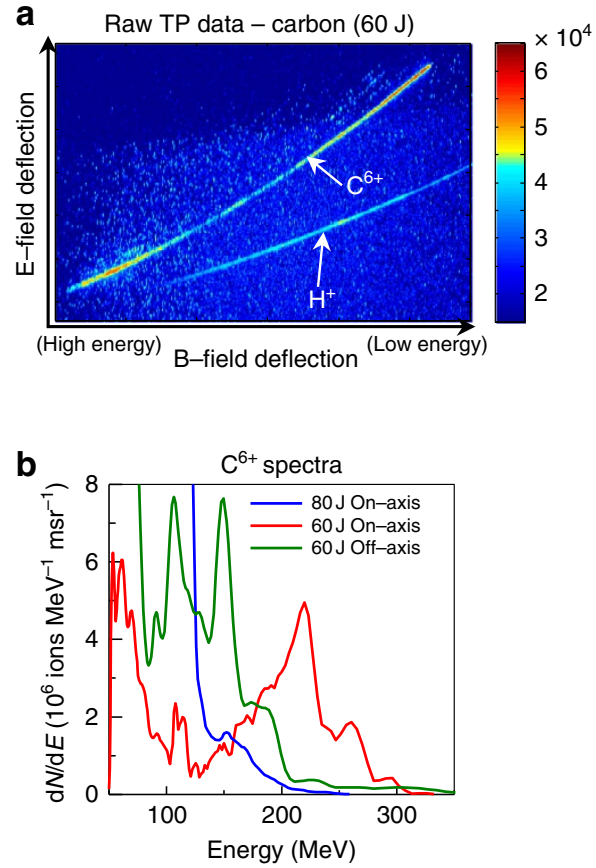
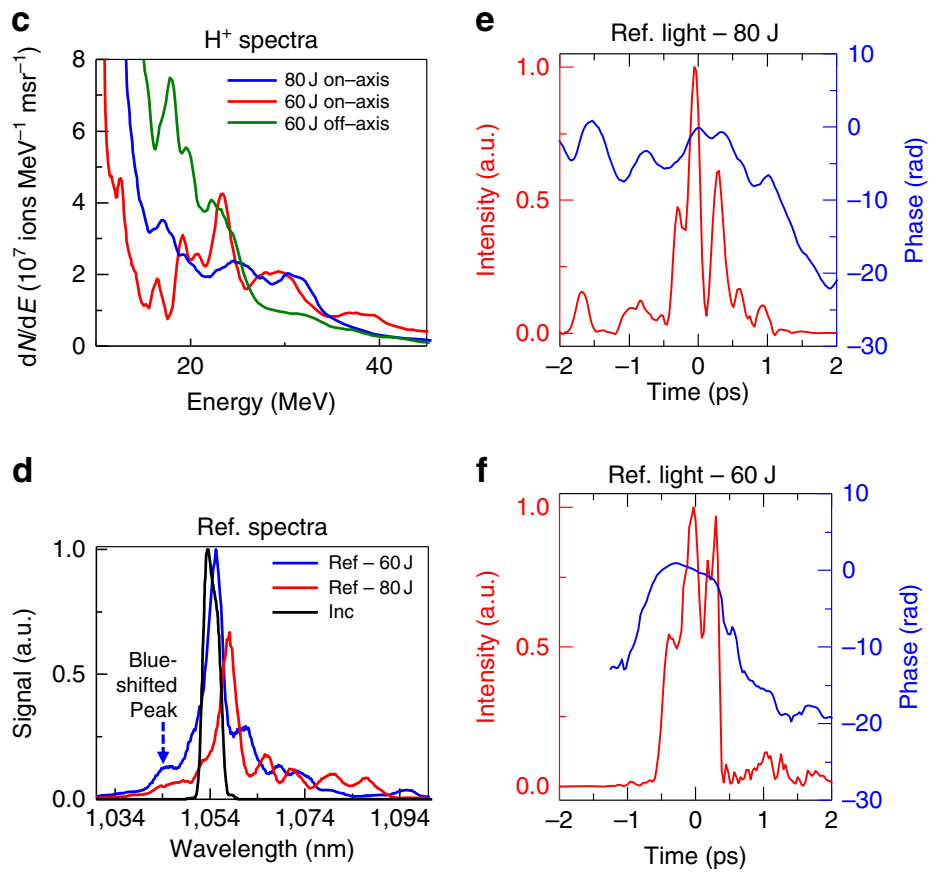

Figure $\mathbf{5}$ | Results from f/1.5 laser focus onto $\mathbf{2 5 0} \mathbf{~ n m ~ s y n t h e t i c ~ d i a m o n d ~ f o i l . ~ ( a ) ~ R a w ~ T P ~ d a t a ~ f r o m ~} 60-\mathrm{J}$ laser shot onto 250 nm diamond foil (Table $1-$ Experiment VII); (b) on-axis $C^{6}+$ spectrum (solid red line) and off-axis $C^{6}+$ spectrum (solid green line) from $60 \mathrm{~J}$ shot (Table $1-$ Experiment VII) showing carbon spectral peaks. On-axis $\mathrm{C}^{6+}$ spectrum (solid blue line) from 80-J shot (Table 1-Experiment VI) with no carbon spectral peak; (c) corresponding on-axis $\mathrm{H}^{+}$spectrum (solid red line) and off-axis $\mathrm{H}^{+}$spectrum (solid green line) from 60-J shot showing proton spectral peaks. On-axis $\mathrm{H}^{+}$spectrum (solid blue line) from 80-J shot with no proton spectral peak; (d) reflected light spectrum from 80-J shot (solid red line) and from 60-J shot (solid blue line) along with incident laser spectrum (solid black line). Reducing the laser intensity leads to blue-shifted reflected laser spectral peak which is indicative of reduced target pre-expansion; (e) time-resolved reflected light intensity (solid red line) and temporal phase (solid blue line) from 80 -J shot with no initial blue-shifted temporal phase indicative of significant target pre-expansion; (f) time-resolved reflected light intensity (solid red line) and temporal phase (dotted blue line) from 60-J shot with strong initial blue-shifted temporal phase indicative of reduced target pre-expansion. 
a
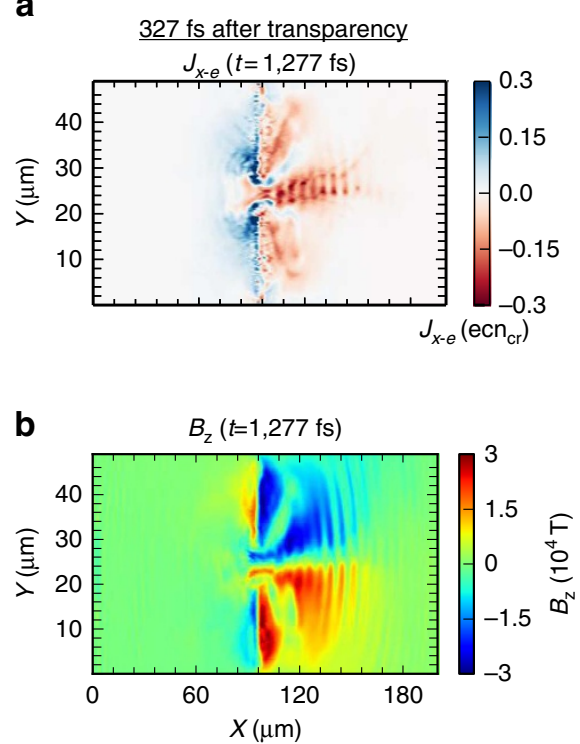

C
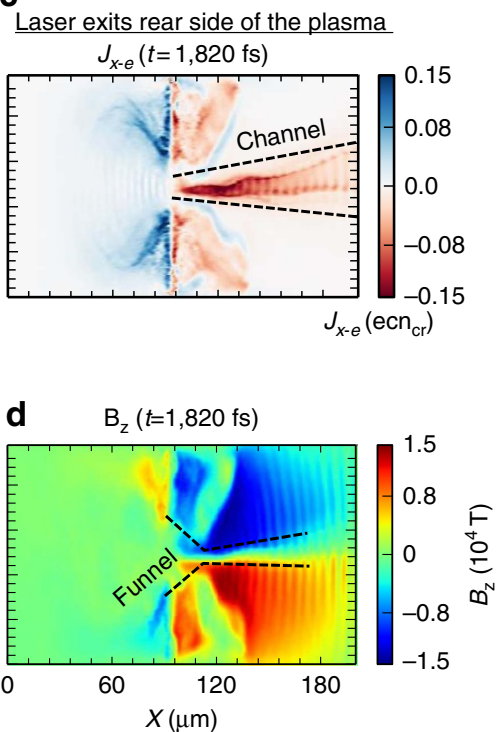

e
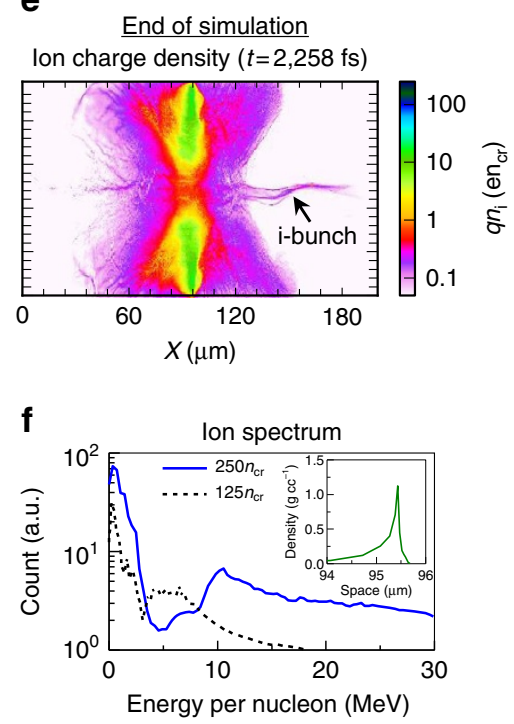

Figure 6 | Overview of 2D VPIC simulation results from $f / 1.5$ laser focus onto pre-expanded 250-nm-thick aluminium foil (a) Longitudinal electron current density $\left(J_{x-\mathrm{e}}\right)$; (b) Self-generated azimuthal magnetic field $\left(B_{\mathrm{z}}\right)$ in the plasma at $t=1,277 \mathrm{fs}$ showing the forward electron jet. Plasma transparency occurs at $950 \mathrm{fs} ;(\mathbf{c})$ Longitudinal electron current density $\left(J_{x-\mathrm{e}}\right)$; (d) Self-generated azimuthal magnetic field $\left(B_{z}\right)$ in the plasma at $t=1,820 \mathrm{fs}$ when the laser exits the rear side of the plasma; (e) Final plasma ion charge density profile at the end of the simulation at $t=2,258 \mathrm{fs}$ with corresponding ion jet; (f) Final ion energy spectrum (solid blue line) with a dominant spectral peak at $10.6 \mathrm{MeV}$ per nucleon; inset shows the pre-expanded plasma profile from 250-nm-thick aluminum foil that was used as input to the VPIC simulation. Dashed black line shows the ion energy spectrum with inferior spectral peak $\sim 5 \mathrm{MeV}$ per nucleon from a simulation of non-optimal onset of relativistic transparency.

Supplementary Figure 6 shows the measured ion energy spectral peak $(\mathrm{MeV} / \mathrm{u})$ as a function of the measured plasma critical surface velocity towards the laser $v_{c s}$ before the onset of RT for a collection of shots. The results show that the ion spectral peaks appear when $v_{c s}>1 \mu \mathrm{mps}^{-1}$. No ion spectral peak is observed when $v_{c s}<1 \mu \mathrm{mps}^{-1}$. Overall, these results indicate that maintaining higher initial plasma density by reducing the foil pre-expansion (thereby having a higher $v_{c s}$ before the onset of RT) and ensuring the onset of RT occurs near the peak of the main laser-pulse are keys to generate ion spectral peaks in our experiments.

Rad-hydro simulations of $250 \mathrm{~nm}$ Al target pre-expansion. Recent study at Trident has shown that the laser pedestal preexpansion of target must be properly accounted and provided as input to the subsequent particle-in-cell (PIC) simulation ${ }^{29}$. We simulate the target pre-expansion of 250-nm-thick aluminium foil (density-2.7 $\mathrm{g} \mathrm{cm}^{-3}$ ) both in $1 \mathrm{D}$ and $2 \mathrm{D}$ using rad-hydro codes HELIOS $^{48}$ and HYDRA ${ }^{47}$, respectively. The resultant 1D (Fig. 6f inset) and 2D plasma profiles (Supplementary Fig. 7) before the arrival of main pulse are exponential at the front and have a sharp drop at the rear side. The HELIOS and HYDRA results are consistent with each other. The peak density is $1.1 \mathrm{~g} \mathrm{~cm}^{-3}$ (that is $250 n_{\mathrm{cr}}$ for $\mathrm{Al}^{11}+$ ionization state). A crucial requirement for the generation of quasi-monoenergetic ion beams in our experiment is the transparency onset timing to be near the peak of the main laser pulse as indicated by the backscattered FROG measurements (Figs 1a and 3e). When the $1 \mathrm{D}$ pre-expanded plasma profile (Fig. $6 \mathrm{f}$ inset) is used as input to the subsequent PIC simulation, the onset of relativistic transparency occurs just $65 \mathrm{fs}$ before the peak of the main laser pulse, consistent with experimental measurements. This qualifies the use of pre-expanded plasma profile from HELIOS as input to the ensuing PIC simulation.
Overview of VPIC Simulations. We performed a series of 2D PIC simulations using the vector-particle-in-cell (VPIC) $\operatorname{code}^{49}$ to investigate the laser-plasma dynamics in the Relativistic Transparency (RT) regime with various initial target densities $\left(125 n_{\mathrm{cr}} \sim 250 n_{\mathrm{cr}}\right)$, charge states $(\mathrm{q} / \mathrm{m}=0.407-0.5$ for heavy ions), composition and electron temperatures ( $1 \mathrm{keV} \sim 32 \mathrm{keV})$, peak laser intensities $\left(2 \sim 8 \times 10^{20} \mathrm{~W} \mathrm{~cm}^{-2}\right)$ and polarizations ( $p$ or $s$ polarization). The collection of simulations shows the formation of a forward-propagating electron jet after the onset of relativistic transparency as a robust feature in this regime. For example, Fig. 6a and Fig. $6 \mathrm{c}$ show the forward electron jet at the rear of the target from the baseline simulation with $32 \mathrm{keV}, 250 n_{\mathrm{cr}}$ target (Fig. $6 \mathrm{f}$ inset), heavy ion charge-to-mass ratio of $0.5, f / 1.5$ laser focus (Intensity- $8 \times 10^{20} \mathrm{~W} \mathrm{~cm}^{-2}$ ), and $p$-polarized laser. Here we use the initial longitudinal density profile of a 250-nm-thick aluminium foil with laser pedestal driven preexpansion from a $1 \mathrm{D}$ rad-hydro simulation, using the HELIOS $\operatorname{code}^{48}$ (see Fig. 6f inset), as input to the VPIC simulation. The initial plasma profile is exponential at the front side and peaks at $x=95.4 \mu \mathrm{m}$ with a peak density of $n_{\mathrm{e}}=250 n_{c r}$. On the rear side, the profile drops sharply in $0.27 \mu \mathrm{m}$ (see Methods for further details). Key laser time markers in the simulation are: laser is launched from the left boundary at $t=0 \mathrm{fs}$; laser reaches target at $t=315 \mathrm{fs}$; onset of transparency occurs at $t=950 \mathrm{fs}$; laser exits the rear side of the plasma at $t=1,785 \mathrm{fs}$; laser exits the right boundary at $t=2,100 \mathrm{fs}$; and the simulations ends at $t=2,258 \mathrm{fs}$. The laser pulse full duration in the simulation is $1,400 \mathrm{fs}$.

The electron jet emerges shortly after the onset of RT, then extends in the forward direction as shown in Fig. $6 \mathrm{a}$ at $327 \mathrm{fs}$ after RT, and finally forms a long channel over $\sim 100 \mu \mathrm{m}$ as the laser exits the plasma (Fig. $6 \mathrm{c}$ for $t=1,820 \mathrm{fs}$ ). The magnitude of the longitudinal current density in the jet is $J_{x-\mathrm{e}} \approx e n_{\mathrm{e}} c \sim e n_{\mathrm{cr}} c$. Assuming a radius comparable to the laser spot size ' $D$ ', the jet carries an enormous current $\sim \pi(D / \lambda)^{2}\left(n_{e} / n_{c r}\right)^{2} I_{\mathrm{A}}$ and the maximum of the associated quasi-static azimuthal magnetic field 
is $\sim(D / \lambda) B_{0}$ at the outer radius of the jet (Fig. 6b,d). Here ' $\lambda$ ' is the laser wavelength, $I_{\mathrm{A}} \approx 17 \mathrm{kA}$ is the Alfvén current and $B_{0}=m c \omega_{0} / e \approx 1.02 \times 10^{4}$ tesla. The magnetic field has the shape of a 'funnel' and it is strongest at $\sim 20-30 \mu \mathrm{m}$ ahead of the initial target position, decreasing gradually further away as the jet diverges. The quasi-static magnetic field persists for $>400 \mathrm{fs}$ after the laser exits the plasma. Supplementary Movie 1 shows the evolution of the self-generated azimuthal magnetic field for the full duration of the VPIC simulation. Although self-generated magnetic fields have been reported in previous studies of laserdriven plasmas ${ }^{39-41,50-55}$, they have not been associated with reducing the ion energy spread as discussed below.

Despite their much smaller charge-to-mass ratio, the plasma ions essentially follow the electron motion and form a similar jet (Fig. 6e). Supplementary Figure 8 shows the corresponding electron-charge density snapshot at the end of the simulation. The ion energy spectrum within the plasma channel at $t=1,820$ fs when the laser exits the rear side of the plasma is still exponential (not shown). Also, we remark for later that the ion jet is chirped in energy along the channel-the ions with the highest (lowest) energies are leading (lagging). By $t=2,258 \mathrm{fs}$, the simulation shows a $10.6 \mathrm{MeV}$ per nucleon ion spectral peak (Fig. $6 \mathrm{f}$ solid blue line) consistent with our experiments. The black arrow in Fig. 6e indicates the location of the $10.6 \mathrm{MeV}$ per nucleon spectral peak in the ion jet. The spectrum in Fig. $6 \mathrm{f}$ also shows another minor ion spectral peak at $1 \mathrm{MeV}$ per nucleon.

The formation of the ion spectral peak as seen in the VPIC simulation is the result of an integrated, self-consistent dynamical process lasting few hundred femtoseconds after the laser exits the rear side of the plasma. To grasp it, we break those dynamics during that period into three separate parts: the electron dynamics, the electrostatic-field dynamics, and the ion dynamics. The magnetic field and the plasma channel that it defines, although part of the self-consistent dynamics, evolve on longer timescales and are therefore considered as given for the sake of understanding.

Late-time electron injection and slow down. We track the electron macroparticles located immediately ahead of the $10.6 \mathrm{MeV}$ per nucleon ion spectral peak near the end of the simulation (Fig. 7d) backward in time. This is important because these electrons are a critical component of the charge density profile inside the plasma channel that leads to the electric field that in turn rotates the ion phase space distribution that shapes the ion peak of interest. The Eulerian fluid velocity vector and the spatial density distribution of the tracked macroparticles are shown for three stages of their motion: the injection at the front side $(x \approx 60-90 \mu \mathrm{m})$, the slow down in the mouth of the magnetic funnel $(x \approx 90-120 \mu \mathrm{m})$ and further slow down of the localized density peak inside the channel (around $x=140 \mu \mathrm{m})$. Representative snapshots of these stages $(t=1,833,1,972$ and 2,140 fs) are shown in Fig. 7b-d (see Supplementary Movie 2).

The injection of electrons is a result of the sheath field at the front side of the target, shown in Fig. 7a for $t=1,821 \mathrm{fs}$, shortly after the laser exits the target. At this stage, these electrons are spatially dispersed but mostly come from the front side of the target (see Fig. $7 \mathrm{~b}$ for their distribution at $t=1,833 \mathrm{fs}$ ). They are accelerated forward to $\sim 1-3 \mathrm{MeV}$ in the sheath potential at the front side of the target and injected into the mouth of the magnetic funnel $(x \approx 90-120 \mu \mathrm{m})$ and further down through the channel $(x>120 \mu \mathrm{m}$; also see Fig. $6 \mathrm{c})$. Those electrons that encounter the large azimuthal magnetic field near the mouth of the funnel $(x \approx 90-120 \mu \mathrm{m})$ become strongly magnetized with their gyro-radii on the order of a few microns.

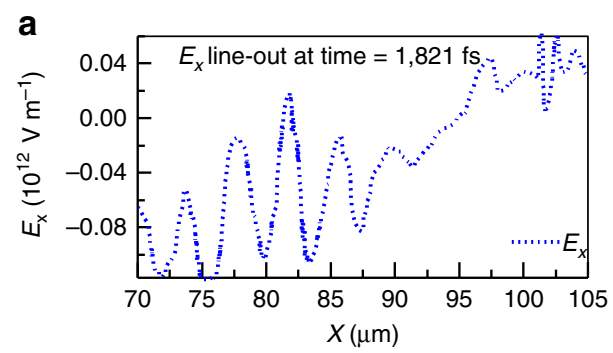

b

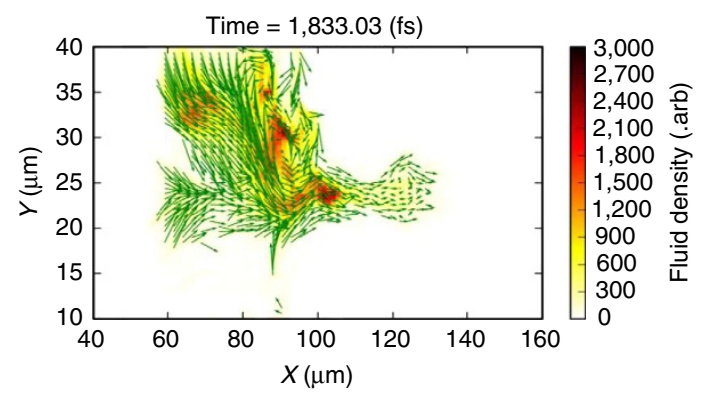

C

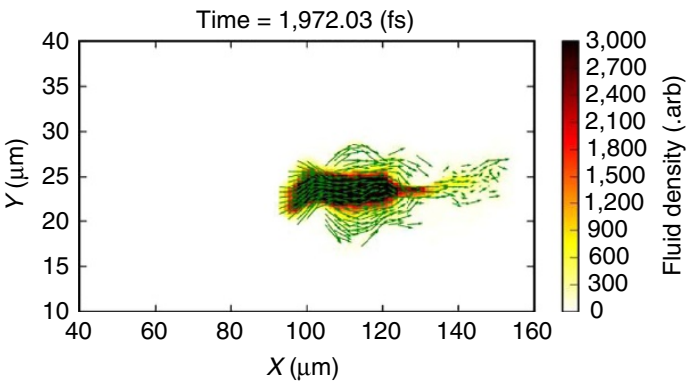

d

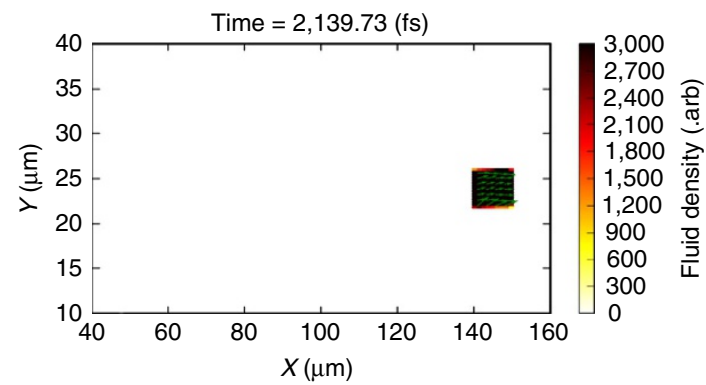

e

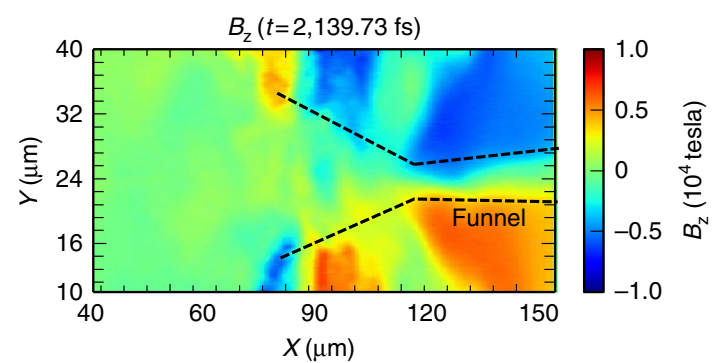

Figure 7 | Electron injection into the plasma channel, and its subsequent slow down after the laser exits the plasma. (a) Longitudinal $E_{x}$ line-out along the middle of the simulation box (averaged over $21 \mu \mathrm{m}<y<27 \mu \mathrm{m}$ ) at $t=1,821$ fs responsible for electron injection into the plasma channel. (b-d) three snapshots of the spatial distribution and the fluid velocity vectors of the tracked electron macroparticles at $t=1,833 \mathrm{fs}, 1,972 \mathrm{fs}$, and $2,140 \mathrm{fs}$, respectively. The length of the fluid velocity vectors is proportional to fluid velocity. The electrons are selected from a rectangular region $140 \mu \mathrm{m}<x<150 \mu \mathrm{m}$ and $21 \mu \mathrm{m}<y<25 \mu \mathrm{m}$ at $t=2,142$ fs just ahead of the ion spectral peak location; (e) self-generated azimuthal magnetic field $\left(B_{z}\right)$ at $t=2,140 \mathrm{fs}$ long after the laser is gone. 
The transverse and the longitudinal magnetic field gradients there significantly slow down the electron fluid drift speed (the cyclotron averaged motion of the ensemble) but not the speed of the individual electrons. Hence, at this compression stage, the slow down of the electron fluid increases the electron density locally ${ }^{56}$. (The electrostatic force plays a negligible role here.) However, this barrier is weakest near the channel axis where the magnetic field and its gradient are smallest and the electrons stream through the channel (Fig. 7c).

In the third stage, these electrons overtake the ions of interest (the ions that will form a spectral peak) and encounter another magnetic barrier, a kink in the channel around $x=135 \mu \mathrm{m}$. Around that point, the electron forward motion slows down and their density increases again. The resulting electrondensity peak causes an electric field labelled ' 3 ' in Fig. 8 (discussed below).

Late-time evolution of the longitudinal electric field. The dynamics described above leads to a double-hump longitudinal electric-field pattern (Fig. 8a) along the plasma channel after the passage of the detached sheath field driven by the laser. Figure $8 \mathrm{~b}$ shows the line-outs of the ion, electron and net charge densities together with the longitudinal electric field along the plasma channel at $t=2,030 \mathrm{fs}$ (marked by dashed black line in Fig. 8a). The first electric-field peak (labelled ' 1 ' in Fig. 8a) results from the slow down of the electron fluid near the 'mouth' of the magnetic 'funnel' increasing the electron density locally around $x=120 \mu \mathrm{m}$ (Fig. 8b). The excess electron-charge density decreases around
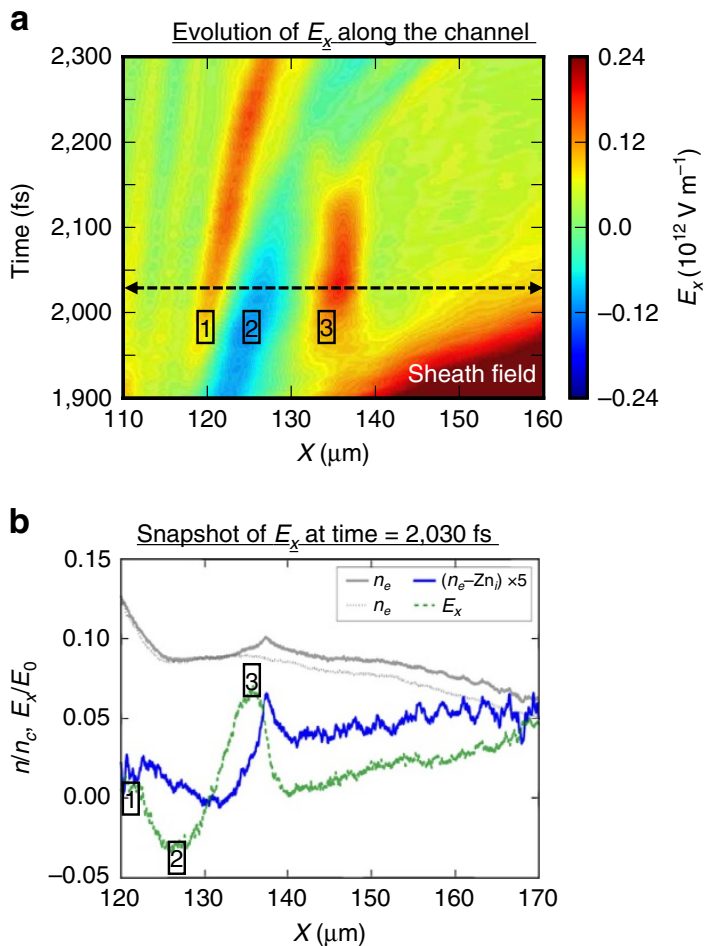

Figure 8 | Longitudinal electric field along the plasma channel responsible for ion energy spread reduction. (a) late-time evolution of the longitudinal electric-field line-out (averaged over $21 \mu \mathrm{m}<y<27 \mu \mathrm{m}$ ) along the channel from $t=1,900$ fs to $t=2,300$ fs after the laser exits the plasma; (b) the electron (solid grey line), ion (dotted grey line), and net charge (solid blue line) densities and the resulting longitudinal electric field (dashed green line) along the plasma channel at $t=2,030$ fs that leads to the particular electric field pattern along the plasma channel. $x=125 \mu \mathrm{m}$ (Fig. $8 \mathrm{~b}$ ) that causes the electric-field valley (labelled ' 2 ' in Fig. 8a).

The near-stationary transient electric-field peak inside the plasma channel between $t \sim 1,950$ fs and $t \sim 2,150$ fs (labelled ' 3 ' in Fig. $8 \mathrm{a}$ around $x=135 \mu \mathrm{m})$ is associated with the nearby electron-density peak (Fig. $8 \mathrm{~b}$ ). It is important to realize that the electron fluid inside the plasma channel is in a continuous motion and it does not stand still anywhere inside the plasma channel as seen in the Supplementary Movie 2. Rather, a decrease in the magnetized electron fluid velocity around $x=135 \mu \mathrm{m}$ increases its density at that position ${ }^{56}$.

The double-hump electric-field pattern is sustained by the continuous injection of electrons from the front side of the plasma. As the injected electron current weakens over time, the magnetic field also weakens and recedes away from the target (see Supplementary Movie 1). The phase velocities of the first peak/valley are synchronous with the phase velocity of the magnetic-field recession, which is a small fraction of the speed of the light and close to the background ion velocity.

Ion spectral peak formation. Figure 9 shows representative trajectories of ions that traverse the plasma channel and the associated late-time electric-field pattern between $t=1,900 \mathrm{fs}$ and $t=2,300 \mathrm{fs}$ and their energy gain/loss. As the ion beam has an energy chirp (increasing from left to right), both the slow-moving and the nearly stationary transient longitudinal electric fields can generate ion spectral peaks. The representative ion with trajectory marked by ' $x$ ' (' $\bullet$ ') in Fig. 9a has lower (higher) initial energy due to the energy chirp and moves synchronously with the first
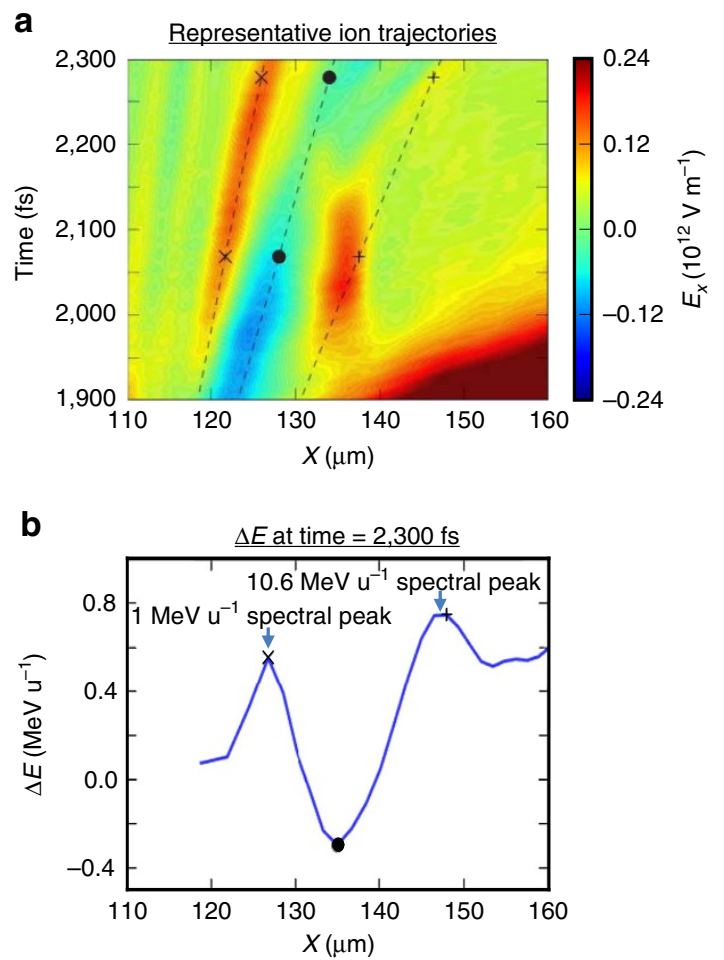

Figure 9 | lon energy spread reduction from the longitudinal electric field after the laser exits the plasma. (a) Three representative ion trajectories (marked by $\times, \bullet,+$ ) overlaid on top of the evolving longitudinal electric field in the plasma channel; (b) energy gain/loss per nucleon at $t=2,300 \mathrm{fs}$ with respect to the ion location in the plasma channel at $t=1,900 \mathrm{fs}$. Also marked is the final energy gain/loss of the representative ions in the spectral peaks. 
electric-field peak (valley). This causes phase space rotation, forming an ion spectral peak. Another spectral peak is formed by the stationary electric field (labelled ' 3 ') as it has finite time duration and is temporally separated from other field patterns (the earlier sheath field that moves away quickly and the field valley, labelled as ' 2 ', that moves in at a later time). The energy gain of the ion is correlated with its arrival time at the location of this transient field. Those fast (slow) ions arrive too early (late) with respect to the field duration do not gain energy, while those ions that arrive at the right time and have transit times across the field smaller than the field duration will gain the same energy. Therefore a spectral peak will form due to the variation in energy gain correlated with the energy chirp of the ions. Figure $9 \mathrm{~b}$ shows the ion energy gain/loss per nucleon between $t=1,900 \mathrm{fs}$ and $t=2,300 \mathrm{fs}$ with respect to their location in the plasma channel. This result shows spectral peaks at $1 \mathrm{MeV}$ per nucleon and $10.6 \mathrm{MeV}$ per nucleon from the slow moving E-fields and from the near-stationary electric field, respectively. The $10.6 \mathrm{MeV}$ per nucleon peak is close to the experimental results. No persistent ion spectral peak at higher energy is formed from the detached sheath field that moves substantially faster than the ions, despite its much larger amplitude.

Our rad-hydro simulation indicates that ' $C$ ' foil expands more than the 'Al' foil during the laser-pulse pedestal for the same laser parameters, resulting in lower initial peak plasma density that leads to non-optimal onset of relativistic transparency and lack of ion spectral peak. This effect is investigated in the simulation with a lower initial plasma density (a peak plasma density of $125 n_{\mathrm{cr}}$ ) but other parameters are kept the same. While the essential processes discussed above are present in this simulation, the onset of relativistic transparency for this target occurred at $270 \mathrm{fs}$ earlier than for the $250 n_{\mathrm{cr}}$ target, leading to weaker electron jet and residual self-generated fields $\left(\sim 1 / 3\right.$ of those in the $250 n_{\mathrm{cr}}$ simulation). The resulting ion energy spectrum (dashed black line in Fig. 6f) shows a less pronounced ion spectral peak at $5 \mathrm{MeV}$ per nucleon. Therefore, it is likely that the target pre-expansion plays an important role both in setting up strong self-generated plasma fields and relativistic transparency onset timing leading to the subsequent ion spectral peak formation.

\section{Discussion}

The question arises as to whether the ion spectral peaking reported here could be due to a different mechanism, such as magnetic vortex acceleration as defined in ref. 42. 'Magnetic vortex acceleration' relies on self-generated quasi-static magnetic field at the rear side of the plasma for efficient forward ion acceleration and collimation $^{39-42}$. In the magnetic vortex mechanism, as explained in ref. 42, magnetic pressure expels electrons from the magnetic region into the plasma channel and builds up an electrostatic field, which accelerates the ions forward at the plasma-vacuum interface $^{42}$. In our case, we also see self-generated quasi-static magnetic field at the rear side of the plasma similar in nature to ref. 42. However, in our case the electrons responsible for reducing the ion energy spread are injected from the front side of the plasma and not from the magnetic field surrounding the plasma channel. Several other concerns and issues such as contribution of holeboring radiation-pressure ion acceleration, tamping of heavy ions by protons, origin of proton spectral peaks, and the relevance to BOA mechanism are discussed in detail under the section 'Supplementary Discussion' in the Supplementary Information.

In summary, we have demonstrated laser-driven ion beams with narrow spectral peaks at energies up to $18 \mathrm{MeV}$ per nucleon and $\sim 5 \%$ conversion efficiency from $0.12 \mathrm{PW}$ laser interactions with planar foils. Computer simulations show a self-organizing scheme that reduces the ion energy spread using self-generated fields from optimized laser-plasma interactions in the relativistic transparency regime. Furthermore, divergence control (collimation/focusing) of these energetic narrow energy spread ion beams is a crucial next step in making these ion beams suitable for various applications ${ }^{57-60}$. Also, a lot of underlying microphysics still remains to be understood in detail. For example, these include identifying the exact relationship between the target pre-expansion and the amount of electron current/associated magnetic field, how exactly the timing on the onset of relativistic transparency affects the ion energy spread reduction, and relevance/consequence of Alfvén limit in relativistic laser-plasmas ${ }^{61}$.

\section{Methods}

Laser system and ion diagnostics. The experiments were conducted at Trident laser facility at Los Alamos National Laboratory, USA. The Trident laser (80 J, $650 \mathrm{fs}$ FWHM, 1,053 nm wavelength, linear polarization) is focused at normal incidence onto the target using an $f / 3$ off-axis parabola (OAP) to a spot size of $10 \mu \mathrm{m}$ diameter (first Airy minimum containing 65\% laser energy) with a peak laser intensity of $2 \times 10^{20} \mathrm{~W} \mathrm{~cm}^{-2}\left(a_{0} \approx 13\right)$. Plasma mirrors were not used in the experiment. A high resolution TP employing $0.91 \mathrm{~T}$ magnetic field over $20-\mathrm{cm}$ long and a pair of copper electrodes, also $20-\mathrm{cm}$ long, charged up to $28 \mathrm{kV}$ potential was used to quantify the resulting ion spectra from the laser-plasma interaction at on-axis

( 0 degrees) and off-axis $(8.5 \text { degrees })^{62}$. Image plates were used as ion detectors in the TP and they were cross-calibrated against CR-39 nuclear track detectors. The instrumental cut-off is at $50 \mathrm{MeV}$ for Aluminium ions due to an $18-\mu \mathrm{m}$-thick aluminium foil placed in front of the ion detector to block laser light. The protons had a low energy cut-off at $9 \mathrm{MeV}$ due to the size of the electrodes and the image plate. For this particular experiment the TP was rotated between on-axis and off-axis for ion spectra characterization. The divergence of the ion beam profile was characterized using a magnetic spectrometer called iWASP (ion wide angle spectrometer) ${ }^{63}$ and image plate detectors. The iWASP was used only on selected shots as it would block the use of any diagnostic located further downstream.

The second set of experiments used a faster $f / 1.5$ OAP to focus the same Trident laser to a spot size of 5 - $\mu \mathrm{m}$ diameter (first Airy minimum containing $65 \%$ laser energy) with a peak laser intensity of $8 \times 10^{20} \mathrm{~W} \mathrm{~cm}^{-2}$. For $f / 1.5$ experiments we developed two additional high-dispersion Thomson parabolas employing longer electrodes (up to $50 \mathrm{~cm}$ ), shorter magnetic field $(0.82$ tesla over

$10-\mathrm{cm}$ long), and longer drift length that enabled better separation of traces with different charge states. The two TPs were located side-by-side on-axis and $11^{\circ}$ off-axis enabling the simultaneous on-axis and off-axis TP data collection (see Supplementary Fig. 4 for more details). The maximum raw signal strength in the TP data reported in this article was $5.7 \times 10^{4}$, which is well below the saturation limit of $6.5 \times 10^{4}$. The ion beam profile in this experiment was measured using a radiochromic film $(25 \times 20 \mathrm{~cm}$ with $1-\mathrm{cm}$ gap in the middle for downstream diagnostics) instead of the iWASP magnetic spectrometer used earlier. The front of the RCF was covered with $75-\mu \mathrm{m}$-thick aluminium foil to block the laser light, aluminium ions up to $180 \mathrm{MeV}$ and protons up to $3 \mathrm{MeV}$. Although we cannot separate the ion and proton beam profiles using the RCF signal in our configuration, we assume they have similar divergence as shown by the earlier iWASP measurements in Fig. 1g.

Conversion efficiency estimation. For example, the conversion efficiency from laser energy to aluminium ions in our experiment is estimated as follows. First, we integrate the aluminium ion energy spectra from the TP in Fig. 1d,e to obtain the aluminium ion yield per unit solid angle at $0^{\circ}$ and $8.5^{\circ}$, respectively. The angularly resolved ion energy spectrum along the horizontal plane with iWASP spectrometer in Fig. $1 \mathrm{~g}$ shows aluminium ions up to $17^{\circ}$ half-width-half-maximum. We integrate the measured ion beam profile along this horizontal slice by using the $0^{\circ}$ and $8.5^{\circ}$ ion yields as anchors to enable an interpolation of the ion yields/solid angle for the full angular distribution. Assuming radial symmetry, the total ion yield is computed from the interpolated angular distribution. Previous measurements at the Trident laser facility in the relativistic transparency regime using diamond targets have shown that the ion yields are almost independent of whether the measurement is made in the horizontal plane or in the vertical plane ${ }^{46}$. On the basis of this result, we expect that the measured aluminium ion spectra in Fig. 1d,e may be assumed to be radially symmetric.

Particle-in-cell simulation. Two-dimensional VPIC ${ }^{49}$ simulations were performed with the Trident laser parameters. The simulation box size is $200 \mu \mathrm{m}$ in $x$ direction (laser propagates from left to right) and $50 \mu \mathrm{m}$ in $y$ direction with an exponential aluminium plasma profile facing the incoming laser. We used 382 cells per wavelength in the $x$ direction and 256 cells per wavelength in the $y$ direction in the simulation. In the nominal simulation discussed in the article, the slab target consists of electrons and fully ionized ions with a charge-to-mass ratio of $0.5 \mathrm{e} / \mathrm{m}$ where $m_{p}$ is the proton mass. This charge-to-mass ratio is the same as $\mathrm{C}^{6+}$ ion and close to the $\mathrm{Al}^{11}+$ ion, the dominant ion species found in the experiments using 
diamond-like carbon and aluminium foil targets, respectively. We used 625 particles per cell for each species in the simulation. Initial target temperatures are $T_{e}=32 \mathrm{keV}$ and $T_{i}=1 \mathrm{keV}$, respectively. The $p$-polarized laser at $1 \mu \mathrm{m}$ wavelength is launched from the left boundary of the box and focused at the peak target density at $x=95.4 \mu \mathrm{m}$. The laser intensity has a temporal profile of $I(t)=I_{\text {peak }} \sin ^{2}(\pi t / 2 \tau)$, where $\tau=650 \mathrm{fs}$ (The laser pulse full duration is $1,400 \mathrm{fs}$ that is $420-\mu \mathrm{m}$ long in free-space). The transverse laser intensity profile is Gaussian with a spot size of $2.5 \mu \mathrm{m}\left(f / 1.5\right.$ laser focus) and the peak intensity is $I_{\text {peak }}=8 \times 10^{20} \mathrm{~W} \mathrm{~cm}^{-2}$ $\left(a_{0}=24\right)$. The particles were injected into the simulation box right before the laser reaches the target front surface to avoid plasma self-expansion.

\section{References}

1. Roth, M. et al. Transport of laser accelerated proton beams and isochoric heating of matter. J. Phys. Conf. Ser. 244, 1-6 (2010).

2. Patel, P. K. et al. Isochoric heating of solid-density matter with an ultrafast proton beam. Phys. Rev. Lett. 91, 125004 (2003).

3. Roth, M. et al. Fast ignition by intense laser-accelerated proton beams. Phys. Rev. Lett. 86, 436-439 (2001).

4. Fernandez, J. C. et al. Fast ignition with laser-driven proton and ion beams. Nucl. Fusion 54, 054006 (2014).

5. Fernandez, J. C. et al. Progress and prospects of ion-driven fast ignition. Nucl. Fusion 49, 065004 (2009).

6. Honrubia, J. J., Fernandez, J. C., Temporal, M., Hegelich, B. M. \& Meyerter-Vehn, J. Fast ignition by laser-driven carbon beams. J. Phys. Conf. Ser. 244, 022038 (2010).

7. Tabak, M. et al. Ignition and high-gain with ultrapowerful lasers. Phys. Plasmas 1, 1626-1634 (1994).

8. Macchi, A., Borghesi, M. \& Passoni, M. Ion acceleration by superintense laser-plasma interaction. Rev. Mod. Phys. 85, 751-793 (2013).

9. Esirkepov, T. Z. et al. Proposed double-layer target for the generation of high-quality laser-accelerated ion beams. Phys. Rev. Lett. 89, 175003 (2002).

10. Schwoerer, H. et al. Laser-plasma acceleration of quasi-monoenergetic protons from microstructured targets. Nature 439, 445-448 (2006).

11. Hegelich, B. M. et al. Laser acceleration of quasi-monoenergetic $\mathrm{MeV}$ ion beams. Nature 439, 441-444 (2006).

12. Robinson, A. P. L., Zepf, M., Kar, S., Evans, R. G. \& Bellei, C. Radiation pressure acceleration of thin foils with circularly polarized laser pulses. New J. Phys. 10, 013021 (2008).

13. Henig, A. et al. Radiation-pressure acceleration of ion beams driven by circularly polarized laser pulses. Phys. Rev. Lett. 103, 245003 (2009).

14. Kar, S. et al. Ion acceleration in multispecies targets driven by intense laser radiation pressure. Phys. Rev. Lett. 109, 185006 (2012).

15. Fiuza, F. et al. Laser-driven shock acceleration of monoenergetic ion beams. Phys. Rev. Lett. 109, 215001 (2012).

16. Haberberger, D. et al. Collisionless shocks in laser-produced plasma generatemonoenergetic high-energy proton beams. Nat. Phys. 8, 95-99 (2011).

17. Palmer, C. A. et al. Monoenergetic proton beams accelerated by a radiation pressure driven shock. Phys. Rev. Lett. 106, 014801 (2011).

18. Dollar, F. et al. Control of energy spread and dark current in proton and ion beams generated in high-contrast laser solid interactions. Phys. Rev. Lett. 107, 065003 (2011).

19. Jung, D. et al. Monoenergetic ion beam generation by driving ion solitary waves with circularly polarized laser light. Phys. Rev. Lett. 107, 115002 (2011).

20. Steinke, S. et al. Stable laser-ion acceleration in the light sail regime. Phys. Rev. ST. Accel. Beams 16, 011303 (2013).

21. Maksimchuk, A., Gu, S., Flippo, K., Umstadter, D. \& Bychenkov, V. Y. Forward ion acceleration in thin films driven by a high-intensity laser. Phys. Rev. Lett. 84, 4108-4111 (2000)

22. Snavely, R. A. et al. Intense high-energy proton beams from petawatt-laser irradiation of solids. Phys. Rev. Lett. 85, 2945-2948 (2000).

23. Hatchett, S. P. et al. Electron, photon, and ion beams from the relativistic interaction of Petawatt laser pulses with solid targets. Phys. Plasmas 7, 2076-2082 (2000).

24. Wilks, S. C. et al. Energetic proton generation in ultra-intense laser-solid interactions. Phys. Plasmas 8, 542-549 (2001).

25. Borghesi, M. et al. Multi-MeV proton source investigations in ultraintense laser-foil interactions. Phys. Rev. Lett. 92, 055003 (2004).

26. Mackinnon, A. J. et al. Effect of plasma scale length on multi-MeV proton production by intense laser pulses. Phys. Rev. Lett. 86, 1769-1772 (2001).

27. Fuchs, J. et al. Laser-driven proton scaling laws and new paths towards energy increase. Nat. Phys. 2, 48-54 (2006).

28. Levy, M. C., Wilks, S. C., Tabak, M., Libby, S. B. \& Baring, M. G. Petawatt laser absorption bounded. Nat. Commun. 5, 4149 (2014).

29. Schollmeier, M. et al. Laser-to-hot-electron conversion limitations in relativistic laser matter interactions due to multi-picosecond dynamics. Phys. Plasmas 22, $043116(2015)$
30. Esirkepov, T., Borghesi, M., Bulanov, S. V., Mourou, G. \& Tajima, T. Highly efficient relativistic-ion generation in the laser-piston regime. Phys. Rev. Lett. 92, 175003 (2004).

31. Kim, I. J. et al. Transition of proton energy scaling using an ultrathin target irradiated by linearly polarized femtosecond laser pulses. Phys. Rev. Lett. 111, 165003 (2013).

32. Yan, X. Q. et al. Generating high-current monoenergetic proton beams by a circularly polarized laser pulse in the phase-stable acceleration regime. Phys. Rev. Lett. 100, 135003 (2008).

33. Qiao, B., Zepf, M., Borghesi, M. \& Geissler, M. Stable GeV ion-beam acceleration from thin foils by circularly polarized laser pulses. Phys. Rev. Lett 102, 145002 (2009).

34. Yan, X. Q., Tajima, T., Hegelich, M., Yin, L. \& Habs, D. Theory of laser ion acceleration from a foil target of nanometer thickness. Appl. Phys. B 98, 711-721 (2010).

35. Tajima, T., Habs, D. \& Yan, X. Laser acceleration of ions for radiation therapy. Rev. Accel. Sci. Technol. 02, 201-228 (2009).

36. Yin, L., Albright, B. J., Bowers, K. J., Jung, D., Fernandez, J. C. \& Hegelich, B. M Three-dimensional dynamics of breakout afterburner ion acceleration using high-contrast short-pulse laser and nanoscale targets. Phys. Rev. Lett. 107, 045003 (2011).

37. Jung, D. et al. Efficient carbon ion beam generation from laser-driven volume acceleration. New J. Phys. 15, 023007 (2013).

38. Henig, A. et al. Enhanced laser-driven ion acceleration in the relativistic transparency regime. Phys. Rev. Lett. 103, 045002 (2009).

39. Bulanov, S. S. et al. Generation of $\mathrm{GeV}$ protons from $1 \mathrm{PW}$ laser interaction with near critical density targets. Phys. Plasmas 17, 043105 (2010).

40. Fukuda, Y. et al. Energy increase in multi-MeV ion acceleration in the interaction of a short pulse laser with a cluster-gas target. Phys. Rev. Lett. 103, 165002 (2009).

41. Nakamura, T., Bulanov, S. V., Esirkepov, T. \& Kando, M. High-energy ions from near-critical density plasmas via magnetic vortex acceleration. Phys. Rev Lett. 105, 135002 (2010).

42. Bulanov, S. V. \& Esirkepov, T. Z. Comment on 'Collimated multi-MeV ion beams from high-intensity laser interactions with underdense plasma'. Phys. Rev. Lett. 98, 049503 (2007).

43. Batha, S. H. et al. TRIDENT high-energy-density facility experimental capabilities and diagnostics. Rev. Sci. Instrum. 79, 10F305 (2008).

44. Palaniyappan, S. et al. Dynamics of relativistic transparency and optical shuttering in expanding overdense plasmas. Nat. Phys. 8, 763-769 (2012).

45. Ammosov, M. V., Delone, N. B. \& Krainov, V. P. Tunneling ionization of complex atoms and of atomic ions in an alternating electric field. Sov. Phys JETP 64, 1191-1194 (1986).

46. Jung, D. et al. Beam profiles of proton and carbon ions in the relativistic transparency regime. New J. Phys. 15, 123035 (2013).

47. Marinak, M. M. et al. Three-dimensional HYDRA simulations of National Ignition Facility targets. Phys. Plasmas 8, 2275-2280 (2001)

48. MacFarlane, J. J., Golovkin, I. E. \& Woodruff, P. R. HELIOS-CR A 1-D radiation-magnetohydrodynamics code with inline atomic kinetics modeling. J. Quant. Spectrosc. Radiat. Transf. 99, 381-397 (2005).

49. Bowers, K. J., Albright, B. J., Yin, L., Bergen, B. \& Kwan, T. J. T. Ultrahigh performance three-dimensional electromagnetic relativistic kinetic plasma simulation. Phys. Plasmas 15, 055703 (2008).

50. Willingale, L. et al. Characterization of high-intensity laser propagation in the relativistic transparent regime through measurements of energetic proton beams. Phys. Rev. Lett. 102, 125002 (2009).

51. Pukhov, A. \& MeyerterVehn, J. Relativistic magnetic self-channeling of light in near-critical plasma: Three-dimensional particle-in-cell simulation. Phys. Rev. Lett. 76, 3975-3978 (1996).

52. Fuchs, J. et al. 'Dynamics of subpicosecond relativistic laser pulse selfchanneling in an underdense preformed plasma'-Reply. Phys. Rev. Lett. 81, 4275-4275 (1998).

53. Borghesi, M. et al. Relativistic channeling of a picosecond laser pulse in a nearcritical preformed plasma. Phys. Rev. Lett. 78, 879-882 (1997).

54. Krygier, A. G., Schumacher, D. W. \& Freeman, R. R. On the origin of super-hot electrons from intense laser interactions with solid targets having moderate scale length preformed plasmas. Phys. Plasmas 21, 023112 (2014).

55. Arefiev, A. V., Robinson, A. P. L. \& Khudik, V. N. Novel aspects of direct laser acceleration of relativistic electrons. J. Plasma Phys. 81, 475810404 (2015).

56. Northrop, T. G. The guiding center approximation to charged particle motion. Ann. Phys. 15, 79-101 (1961).

57. Toncian, T. et al. Ultrafast laser-driven microlens to focus and energy-select mega-electron volt protons. Science 312, 410-413 (2006).

58. Bartal, T. et al. Focusing of short-pulse high-intensity laser-accelerated proton beams. Nat. Phys. 8, 139-142 (2011).

59. Cowan, T. E. et al. Ultralow emittance, multi-MeV proton beams from a laser virtual-cathode plasma accelerator. Phys. Rev. Lett. 92, 204801 (2004). 
60. Schollmeier, M. et al. Controlled transport and focusing of laser-accelerated protons with miniature magnetic devices. Phys. Rev. Lett. 101, 055004 (2008).

61. Davies, J. R. The Alfven limit revisited and its relevance to laser-plasma interactions. Laser Part. Beams 24, 299-310 (2006).

62. Jung, D. et al. Development of a high resolution and high dispersion Thomson parabola. Rev. Sci. Instrum. 82, 013306 (2011).

63. Jung, D. et al. A novel high resolution ion wide angle spectrometer. Rev. Sci. Instrum. 82, 043301 (2011).

\section{Acknowledgements}

We gratefully acknowledge the support of the Los Alamos National Laboratory LDRD (Laboratory Directed Research and Development) program (projects 20120721ECR, 20140483ER, and 20140029DR) for this work. We also thank the capable and dedicated Trident laser personnel: R. P. Johnson, T. Shimada, R, Gonzales, S. Reid and R. Mortensen for the laser operation. The simulations were run using LANL Institutional Computing and ASC Capability Computing Campaign allocations. We thank L.Yin and B. Albright for their help with VPIC simulations. S.P thanks J. Schreiber for the thoughtful discussions.

\section{Author contributions}

S.P., D.C.G., R.C.S. and J.C.F. designed and executed the experiment. C.E.H., M.A.S. and C.K. made the targets. S.P. analysed the experimental data. C.H. performed the VPIC simulations. A.B.S. performed HYDRA simulations. S.P. and C.H. wrote the paper with contributions from all the co-authors.

\section{Additional information}

Supplementary Information accompanies this paper at http://www.nature.com/ naturecommunications

Competing financial interests: The authors declare no competing financial interests.

Reprints and permission information is available online at http://npg.nature.com/ reprintsandpermissions/

How to cite this article: Palaniyappan, S. et al. Efficient quasi-monoenergetic ion beams from laser-driven relativistic plasmas. Nat. Commun. 6:10170 doi: 10.1038/ ncomms10170 (2015)

\section{(c) (i)}

This work is licensed under a Creative Commons Attribution 4.0 International License. The images or other third party material in this article are included in the article's Creative Commons license, unless indicated otherwise in the credit line; if the material is not included under the Creative Commons license, users will need to obtain permission from the license holder to reproduce the material. To view a copy of this license, visit http://creativecommons.org/licenses/by/4.0/ 